\title{
1 Interacting grassland species under threat of multiple global change drivers
}

2 Running title: Communities under threat of global change

3

H. De Kort ${ }^{1,2}$, J.G. Prunier ${ }^{1}$, M. Tessier ${ }^{3}$, C. Turlure $^{4}$, M. Baguette ${ }^{1,5}$, V.M. Stevens ${ }^{1}$

1 Station d'Ecologie Théorique et Expérimentale (UMR 5321 SETE), National Center for Scientific

7 Research (CNRS) and Université Toulouse III - Paul Sabatier, 2 Route du CNRS, 09200 Moulis, France.

82 Plant Conservation and Population Biology. Department of Biology, KU Leuven. Kasteelpark Arenberg

931,3001 Leuven, Belgium.

3 Marc Tessier, 62 chemin Del prat, 31320 Auzeville-Tolosane, France.

4 Earth and Life Institute, Université catholique de Louvain, Croix du Sud 4, 1348 Louvain-la-Neuve, Belgium.

5 Institut de Systématique, Evolution, Biodiversité (UMR 7205), Muséum National d'Histoire Naturelle (Sorbonne Universites), CP 50, 45 rue Buffon, 75005 Paris, France.

\section{* Correspondence to: hanne.dekort@kuleuven.be}

Acknowledgments: We thank Gaëlle Blanvillain, Sophie Dardenne, and many students for field assistance, and Murielle Richard for lab assistance. Annie Ouin (INRA), Thomas Houet (CNRS), and the Parc Naturel Régional Pyréénes Ariégeoises (Yannick Barascud and Julien Aït El Mekki) provided the GIS resources used for mapping levels of habitat fragmentation. Primary financial support was provided by the ANR GEMS \& INDHET (ANR-13-JSV7-0010-01 and ANR-12 -BSV7-0023-02). HDK, VMS, $J P$ and MB are members of the Excellence Lab TULIP (ANR-10-LABX-41).

Data accessibility statement: SNP allele frequencies and flanking sequences are archived at Dryad (doi:10.5061/dryad.f371754). 


\section{ABSTRACT}

Aims Multiple environmental changes simultaneously altering the biotic and abiotic context of species are threatening communities and ecosystems worldwide. Exploration and mitigation of the ecoevolutionary impacts of global change threats correspondingly are major components of conservation research, yet joint global change impacts remain poorly studied. Moreover, changes in the biotic context of species are rarely considered when assessing global change induced range shifts. We aim to unravel the contributions of habitat fragmentation, climate warming, genetic variation and biotic interactions to the past, current and future distribution of a rare grassland butterfly.

\section{Location French Pyrenees}

\section{Taxon Phengaris (Maculinea) alcon, Gentiana pneumonanthe}

Methods We examined the combined effects of habitat fragmentation and climate warming on the expected distribution of a specialized grassland butterfly and its host plant species using ecological niche modelling and genetic analysis. More specifically, circuit theory and maximum entropy modelling were used to assess changes in connectivity and habitat suitability under various land use and climate warming scenarios. Complementary, we used pooled RAD sequencing to assess relations between genetic diversity on the one hand, and connectivity, habitat suitability and altitude on the other hand.

Results We show that both habitat fragmentation and climate warming reduce the amount of suitable and reachable habitat. Complete abandonment of mild grazing practices would drastically reshuffle the distribution of suitable habitat and would render most of the remaining suitable patches poorly reachable. Moreover, serial genetic founder effects observed in high-altitudinal populations of both species suggest that their dispersal rates lag behind environmental change.

Main conclusions Together, these findings corroborate the notion that habitat fragmentation, through abandonment of mild grazing practices and agricultural intensification, is the most eminent threat to the highly biodiverse semi-natural grassland ecosystems across Europe. Fighting habitat fragmentation would increase the genetic and ecological resilience of communities toward other global environmental threats.

Key-words: Phengaris (Maculinea) alcon, Gentiana pneumonanthe, ecological niche modelling, connectivity, habitat fragmentation, land use, land conversion, global change, intensification 
INTRODUCTION

Assessing global change-induced shifts in suitable habitat is a major component of species and ecosystem conservation research (Hill et al., 2016; Ettinger \& HilleRisLambers, 2017). Extensively managed landscapes such as seasonally occupied grasslands are renowned for their high species richness, and systematic abandonment of these landscapes is causing pronounced species' declines across Europe, urging for targeted conservation of these valuable ecosystems (Queiroz et al., 2014; Sutcliffe et al., 2015). Ecological niche modelling (ENM) has been widely used to identify the potential distribution of species of conservation concern, and to extrapolate niche-environment relations to likely global change scenarios (Weber et al., 2017). ENM studies have frequently provided interesting insights into the abiotic factors driving species' presence-absence patterns, yet are generally limited to single species. Biotic interactions including parasitism, animal-mediated pollination and predation are, however, crucial for meta-population functioning, and consequently the potential of populations to survive global changes (Wilmers \& Post, 2006; Gilman et al., 2010; Bailey et al., 2014; Godsoe et al., 2017). Especially where multiple global changes act in concert, many species may be facing persistence challenges beyond current acknowledgement (Sirami et al., 2017). An important avenue in global change ecology and conservation biology therefore exists in unravelling the integrated impact of multiple global environmental changes and species' interactions on the distribution and abundance of species (Sirami et al., 2017; Godsoe et al., 2017).

Maximizing our ability to obtain realistic distribution forecasts thus requires an understanding of the multiple factors affecting species in both their abiotic and biotic environment. Biotic interactions are key to natural communities, and their intensity and frequency are shaped by complex eco-evolutionary dynamics within and among species, including gene flow, species sorting, local adaptation and coevolution (Blois et al., 2013). Global changes have been shown to frequently disrupt these dynamics, consequently destabilizing species' interactions and endangering the species involved in ecological networks (Memmott et al., 2007; Tylianakis et al., 2008; Blois et al., 2013; Strona et al. 2016). Species with specific biotic or abiotic requirements and limited dispersal abilities in particular are facing strong declines with increased habitat fragmentation and climate warming (Warren et al., 2001; Clavel et al., 2011; Eskildsen et al., 2015). Moreover, presence-absence data of species living in close association with rare species through shared habitat requirements or direct species' interactions may improve ENM accuracy by increasing the amount of spatial data that can be used to narrow down the realized niche. Despite global conservation priorities toward rare and specialist species, ENMs accounting for species' interactions are, however, rare (González-Salazar et al., 2013; Anderson, 2017).

Ecological niche models can be used in parallel with genetic analysis to inform about the ability of populations and communities to cope with future environmental conditions, as genetically impoverished populations are unlikely to survive the increased stress levels associated with distribution-wide changes in biotic and abiotic conditions (Reed \& Frankham, 2003; Carlson et al., 2014; Kittelson et al., 2015; Ikeda et al., 2017). Reduced habitat suitability due to global environmental 
changes can compromise the genetic diversity, fitness and adaptive potential of natural communities through reducing population sizes and impeding dispersal. Moreover, genetic changes accumulated through past population and community responses to historical environmental shifts can inform forecasts of species distributions in response to future environmental changes (Fordham et al., 2014). Whereas the role of population-level processes in community and ecosystem dynamics is widely acknowledged (Hughes et al., 2008; Fridley \& Grime, 2010; Prieto et al., 2015; Fernandez-Conradi et al., 2017), the relation between population genetic diversity, species' interactions and range dynamics remains poorly studied.

The highly specialized parasitic butterfly Phengaris (=Maculinea) alcon (Alcon blue, hereafter "Alcon"), faces extinctions across its Eurasian range (Wynhoff, 1998). Alcon requires the simultaneous presence of multiple species, including the rare plant Gentiana pneumonanthe (Marsh gentian, hereafter "Gentian"), and an ant of the genus Myrmica (hereafter "Myrmica"). While Myrmica ants are widespread across Eurasia, the Gentian plants have a scattered distribution, restricted to marshes and wet grasslands from northern Portugal and the Balkans in the south to southern Finland in the north (Simmonds, 1946), and strongly depend on grazers for the dispersal of their seeds. Thus, Alcon habitat is expected to be mainly shaped by direct and Gentian-mediated effects of changes in land use and climate. Accordingly, habitat fragmentation, through affecting the regional distribution of its host plant, is believed to be an important driver of Alcon fall backs (Mouquet et al., 2005; Vanden Broeck et al., 2017). While both Alcon and Gentian have been in decline across their range for decennia (IUCN, 1990 and OosterMeijer et al., 1992, resp.), recent declines have been reported in Belgium (e.g. Maes et al., 2004), the Netherlands (e.g. WallisDeVries, 2004), Germany (e.g. Habel et al., 2007), and France (e.g. Tessier, 2015).

The goal of this study is to assess how climate change, habitat fragmentation, and changes in host plant distribution independently and interactively shape past, current, and future distribution of Alcon butterflies in a mountainous area. We focused on the French Pyrenean department of Ariège, featured by seasonal grazing of high-altitudinal semi-natural grasslands. Alcon experiences notable declines in the region, presumably driven by the joint impacts of (spontaneous) reforestation and climate change on Alcon community dynamics. We used ecological niche modelling to map the current, past and future Alcon distribution based on host plant distribution, land use and forest cover, bioclimatic variables, topography, and observed field temperatures. In addition, genetic diversity of Alcon populations was examined in relation to habitat suitability, connectivity, altitude and host plant genetic diversity to obtain realistic prospects of regional Alcon meta-community dynamics.

Specifically, we addressed the following key questions: (i) How do climate warming and land use change interactively impact the amount of habitat suitable for Alcon and its host plant? (ii) Can the predicted host plant distribution be used to enhance Alcon distribution modelling? (iii) How do climate warming and land use change affect the connectivity between suitable habitat patches given the dispersal abilities of Alcon? (iv) Is within-population genetic diversity driven by habitat suitability and connectivity? And (v) can we find new populations based on ecological niche modelling outcomes? 


\section{Study system}

139

140

141

142

143

144

145

146

147

148

149

150

151

152

153

154

The Alcon butterfly requires the simultaneous presence of Gentian plants and Myrmica ants (locally M. scabrinodis). Alcon deposits its eggs on Gentian flower buds, followed by larval predation of the seeds after hatching. After the third moult, the caterpillars drop on the ground, aiming to be found by a Myrmica ant worker. Co-evolved surface chemistry allows the relatively big Alcon caterpillars to be recognized and fed as superior Myrmica larvae (Nash et al., 2008).

A total of 35 Alcon populations (incl. eight recently extinct populations) and 168 Gentian populations were identified after thorough screening of Ariège $\left(4890 \mathrm{~km}^{2}\right)$ (Tessier, 2015). The Alcon sites are structured into four geographical clusters, including two nearby clusters in the Valley Bottoms ecoregion of Ariège and two relatively distant clusters in the Mountain ecoregion (Fig.1, Table S1). The populations span an altitudinal gradient from 400 to $1017 \mathrm{~m}$ (Table S1). Daily capture-mark-recapture surveys covering the full flying season from late June to early September (2014) showed regular dispersal within $3 \mathrm{~km}$ ranges and rare dispersal events up to distances of $17 \mathrm{~km}$ where the landscape is connected through pastural and (semi-)natural grasslands (Fig.S1). In each Alcon population, an Alcon leg and a Gentian leaf per individual were sampled from an average of 26 and 31 individuals, respectively, for DNA extraction and genetic analysis (Table S1).

Predicted Alcon distribution based on temperature, topography, forest cover, land use and host plant presence probabilities.
Predicted Alcon distribution based on temperature, topography, forest cover and land use.
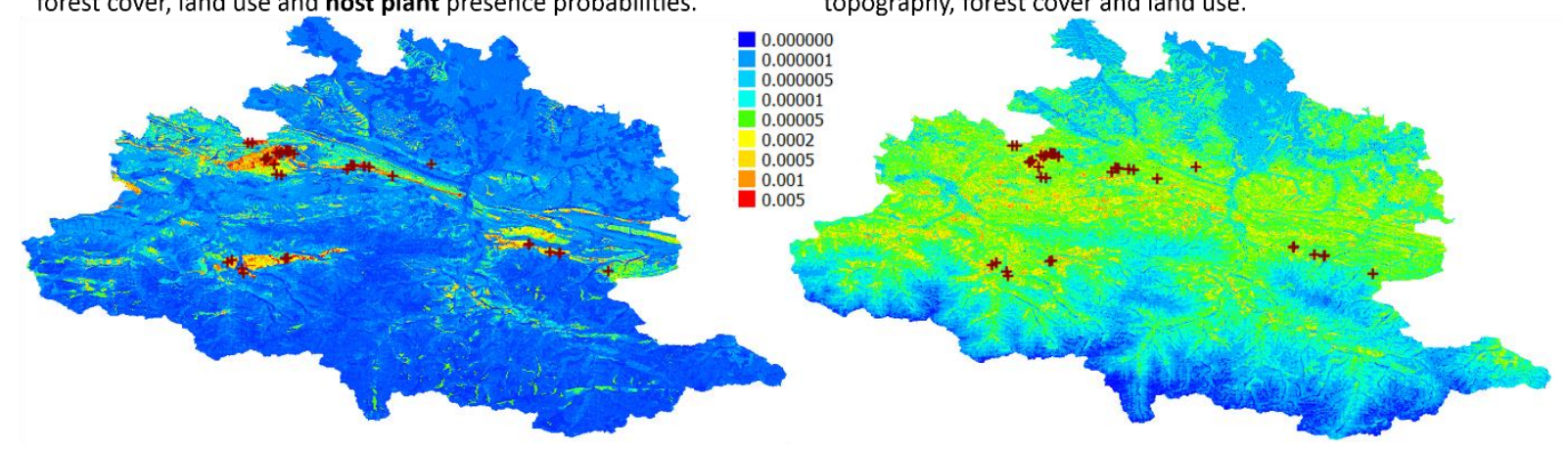

Fig.1. Present habitat suitability map of Alcon with (left) and without (right) accounting for host plant distribution. Colours follow MAXENT raw (relative) presence probability distributions. Yellow to red colours ( $\geq$ suitability threshold of $2 \times 10^{-3}$ ) are deemed suitable for Alcon. Red crosses represent Alcon sampling sites.

\section{Ecological Niche Modelling}

Relations between environmental variables and current species occurrences can be extrapolated to reconstruct past and predict future distributions. We correspondingly used ecological niche modelling 
to map relations between various environmental variables (topography, field temperature, climate, forest cover, land cover, and geological information) and Alcon and Gentian presence. To take into account topographical and microclimatic influences on climate, including cold air drainage, solar irradiation and forest cover, we assessed the relation between observed temperatures, and topography and forest cover. To this end, two temperature loggers (ca. 100 meters apart) were installed at each site to monitor temperature hourly during a year. Observed local monthly minimum temperatures (unaffected by monitor overheating) were extracted (Table S3) and regressed against (i) forest cover and a set of four topographical variables (30 m resolution; Table S2), and (ii) regional monthly minimum temperatures extracted from worldclim.org (v1.4) (ca. 1000m resolution) (Hijmans et al., 2005).

From these twelve monthly temperature models, we selected the two months of April and July based on their ecological relevance (onset of host plant growing season and Alcon flying season, resp.), and the high adjusted $\mathrm{R}^{2}$ values of the underlying regression models (Table S4). Model coefficient estimates of the significant variables were applied to the forest cover, topography and resampled regional temperature maps to obtain a $30 \mathrm{~m}$ resolution temperature map for each of the two months: "APR Topo" and "JUL Topo" (please see Appendix for map construction details). In addition to these topographically corrected temperature variables, a set of ecologically relevant bioclimatic ("bioclim") variables were retrieved from worldclim.org (Table S2, Table S5). Finally, a land use ("Landuse") and a geology (“Geology") map were converted to a $30 \mathrm{~m}$ resolution raster (Table S2). All abiotic maps were used for ENM following the methodology described below.

Four different sets of the environmental variables described above (TEMP topo, BIOCLIM, TOPO, BIOTOPO: Fig.2, Table S2) were used and mutually compared to estimate the present Gentian and Alcon distribution. To reconstruct past distributions (present to Last Glacial Maximum), the TEMP $P_{\text {topo }}$ maps were cooled by 1 to $8^{\circ} \mathrm{C}(\mathrm{C} 1-\mathrm{C} 8)$ while future climate warming was simulated through an increase in temperature of 1 to $3^{\circ} \mathrm{C}$ (W1-W3). Increasing fragmentation of suitable habitat was simulated through (i) partial (30\%) conversion of pastures into forest, (ii) full conversion of pastures into forest, and (iii) full conversion of pastures (intensive grazing) and semi-natural grasslands (extensive seasonal grazing) in the land use map, corresponding to ca. 3\%, 10\% and 13\% reforestation across the study region, respectively (F1-F3). Land conversion and warming were combined to assess the joint impacts of the two global change effects. For the Alcon scenarios, Gentian distribution probabilities retrieved from the corresponding ENMs were included as an additional predictor variable (Fig.2). Myrmica was not used for ENM because it is much more common across the landscape than Gentian.

In addition to the warming and land conversion scenarios depicted above, we modelled a "2100" scenario representing the most likely land conversion and warming conditions predicted for the near future (the year 2100). This scenario was based on historical land use trends and socio-economic drivers in the French Pyrenees (Vacquie et al., 2015), and mild global mean temperature change projected by the low emission scenario (RCP2.6) presented by the IPCC (IPCC, Collins et al., 2013). More specifically, we modelled a modest temperature increase of $+1^{\circ} \mathrm{C}(\mathrm{W} 1)$ and assumed a socio-economic 
context with features of a business-as-usual model (no major changes in global economy, land management and public policy, $17.8 \%$ land abandonment) and with characteristics of a regional cooperation model (implementing ambitious conservation policies to reduce the environmental impact of agro-pastoral activities while optimizing production rates, 3.3\% land abandonment) (Vacquie et al., 2015). We generated a simple approximation of the 2100 socio-economic model through conversion of the $50 \%$ smallest natural and semi-natural grasslands into forest while conserving all existing pastures (ca. 7\% reforestation).
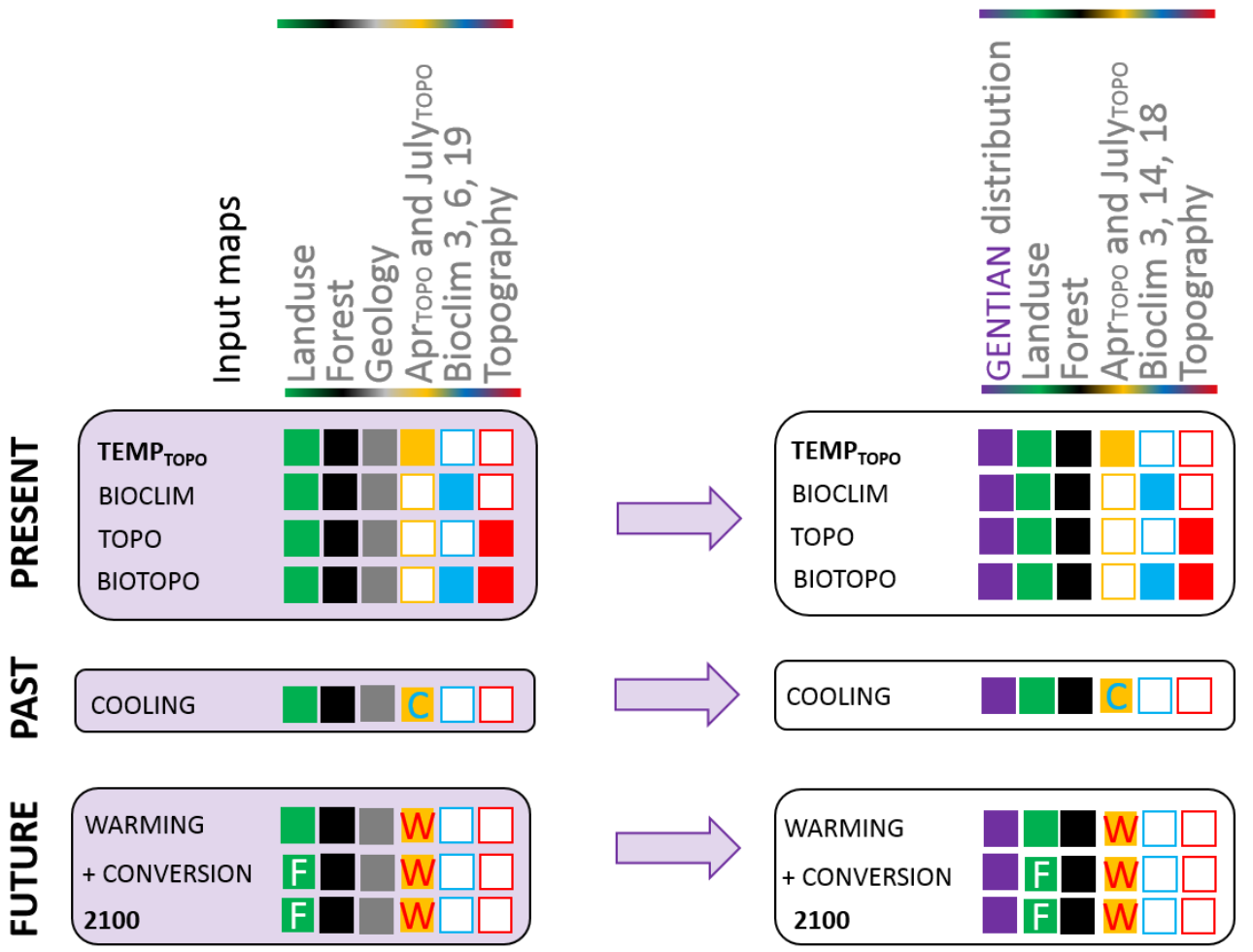

Fig.2. Diagram showing how environmental variables (Input maps) were combined to parameterize ENM for Gentian and Alcon in different scenarios, to obtain predictions on their probable distribution in Ariège. The first four PRESENT scenarios represent the four sets of environmental variables mutually compared for their predictive abilities. The TEMP topo scenario served as the basis for the past and future scenarios, as it had high predictive power and allowed modelling of temperature. Only coloured squares were used in the respective scenarios. Letters in squares refer to the level of alteration modelled relative to the initial conditions of present scenario TEMPтopo. $\mathrm{C}:-1^{\circ} \mathrm{C}$ to $-8^{\circ} \mathrm{C} ; \mathrm{W}:+1^{\circ} \mathrm{C}$ to $+3^{\circ} \mathrm{C} ; \mathrm{F}:+3 \%,+10 \%$ or $+13 \%$ land conversion. Topography (input map) represents four different topographical variables as detailed in Table S2. Note that for future scenarios we always included at least $1^{\circ} \mathrm{C}$ warming because ongoing warming cannot be fully prevented while regional and local policies and land management can, to some extent, mitigate ongoing land conversion.

Habitat suitability maps were generated using maximum entropy modelling with MAXENT v3.3.3 (Phillips et al., 2004; Phillips \& Dudík, 2008), for Gentians and Alcons. MAXENT extracts a random sample of background locations from a user-defined landscape and contrasts these background locations to a list of presence locations in function of the input environmental variables, resulting in a relative occurrence rate (ROR). The ROR describes the relative probability that a grid cell on the 
landscape is part of the collection of presence samples, and represents raw output as recommended over logistic regression (e.g. Merow \& Silander 2015; Halvorsen et al. 2015). The identification of presence data across Ariège took place by visiting a total of 184 hill tops that allowed a clear overview of the surrounding area (Tessier, 2012). To minimize sampling bias that could otherwise result in inaccurate projections and overestimated predictive power (Merow et al., 2013), a buffer area (10km intersection) was drawn around each visiting site, and all environmental input maps were cropped to this buffer (Fig.S4). MAXENT default parameters (500 iterations, regularization multiplier of 1 , convergence threshold of 0.00001 , and 10.000 random background points) were used. Crossvalidation with ten replicates was performed to evaluate prediction uncertainty. Average model performance was assessed through the area under the curve (AUC), with values ranging from 0.5 for a random prediction to 1 for a perfect prediction (Raes \& ter Steege, 2007). The fitted ecological niche model was then projected (i) to the full extent of Ariège to assess the present distribution, and (ii) to the warming, land conversion and cooling scenarios to assess past and future distributions.

Although we limited overfitting through limiting the number of variables to the most ecologically relevant ones, we assessed the additional effect of various beta regularization parameters (a penalty to reduce overfitting) using the Wallace R package (Kass et al. 2018). For both species, the default regularization multiplier in Maxent (1) was comparable to, or exceeded, most other values in both species in terms of AUC and AICc (Table S6, Fig.S5-S6), and was therefore used for all models.

\section{Connectivity and habitat fragmentation}

242 To assess the connectivity between pairs of suitable patches, we used CIRCUITSCAPE (McRae et al., 2013), a circuit theory-based approach that calculates the dispersal resistance experienced at each pixel in a raster landscape based on environmental input maps scored according to presumed dispersal efficiency. Assigning a high score to specific land use features thus results in high dispersal resistance and low connectivity at corresponding locations. We used the Alcon habitat suitability maps resulting from the ENM scenarios as input maps, assuming that high habitat suitability results in more efficient dispersal across the landscape. To avoid overly extensive calculation times, we applied a centroidbased approach rather than a patch approach, and only centroid pairs less than $20 \mathrm{~km}$ (exceeding observed dispersal distances) apart were considered. Suitable habitat patches for centroid determination were delineated using a habitat suitability threshold of $2 \times 10^{-3}$, corresponding to the minimum habitat suitability (provided by ENM) at sampled Alcon sites. Small patches $(<1$ ha) that are unlikely to sustain suitable host plant populations were removed to limit the number of centroids. The effects of climate and land use change on levels of habitat fragmentation were quantified across scenarios using a variety of metrics provided by FRAGSTATS 4.2 (McGarigal et al., 2002) (Fig.3). Connectivity in each scenario was calculated as $\left[(1 / \text { resistance })^{*} 10^{6}\right]$ averaged across all centroids

257 (Tables S7-S8).

Part of the suitable patches is expected to be inaccessible due to low connectivity. Based on observed dispersal events during the CMR survey, a connectivity threshold was assigned beyond which Alcon dispersal is highly unlikely. This threshold corresponded to the connectivity between the two sites with 
the maximum observed dispersal distance $(17 \mathrm{~km}$, between NE2 and NW16), calculated using a CIRCUITSCAPE analysis involving the sampled Alcon populations. The R-package "igraph" was used to identify centroids disconnected from all centroids of the preceding scenario (e.g. for scenario W2, the centroids of W1 were considered as "emigration" sites from which butterflies could depart during the transition from $\mathrm{W} 1$ to $\mathrm{W} 2$ ).

\section{In situ inspection of the ENM}

Although the Ariège department already had been profoundly screened for the presence of Alcon through field surveys by regional naturalists (Tessier 2012, 2015), a selection of 30 coordinates was extracted from the Alcon TEMP TOPO $_{\text {map }}$ based on promising presence probabilities with the twofold aim of (i) discovering new Alcon and Gentian sites and (ii) validating the MAXENT model in situ. Presence probabilities at these "checkpoint" coordinates was statistically compared with probabilities at existing sites and at potentially newly discovered sites. The number of flowering Gentians and the proportion of Gentians with Alcon eggs were counted.

\section{Genetic diversity (GD)}

Stable and/or connected populations typically maintain relatively high genetic diversity. Prior to genetic analysis, extracted DNA was subjected to pooled RADsequencing, resulting in allele frequency (AF) estimates for 478 and 184 high-quality SNPs for Alcons and Gentians, respectively (Fig.S7) (see Appendix for a detailed protocol). To obtain a measure of GD, expected heterozygosity (Nei et al., 1975) was calculated as $2 A F(1-A F)$, averaged across loci, for each sampled population based on the AF estimates (Fig.S12). We subsequently applied regression models to assess the effect of Gentian GD, altitude, habitat suitability and connectivity on Alcon GD. Local habitat suitability was defined as the mean habitat suitability (provided by ENM) in a buffer area of $5 \mathrm{~km}^{2}$ around each successfully sequenced population ( $n=22$; Table S1). Site connectivity was calculated as the average pairwise connectivity between a site and all other sites in the region (Table S1). Altitude was included as a covariate to take into account a potential historical colonization event. In addition, Gentian GD was added to find species' interactions on the level of GD that could be due to shared habitat and/or historical features. Model selection was performed using the Dredge function in R (package "MuMIn").

RESULTS

The four sets of environmental variables used to estimate present Alcon distribution rendered similarly high predictive abilities (AUC) ranging between 0.972 (TOPO) and 0.978 (BIOCLIM). Ignoring host plant distribution resulted in lower AUC values (0.794-0.809, Fig.S9). The topoclimate set (TEMP topo, Table S2), which served as the basis for the various global change predictions, resulted in an AUC of 0.975 . Although these very high values must be interpreted with caution due to the limited spatial scale of the study, the resulting distribution maps provided a realistic representation of the present Alcon 
301

distribution in Ariège (Fig.1), with an estimated $3.44 \%$ of the landscape potentially suitable for the species. A small proportion of this suitable area, at higher altitudes, is unreachable under present environmental conditions (Fig.3).
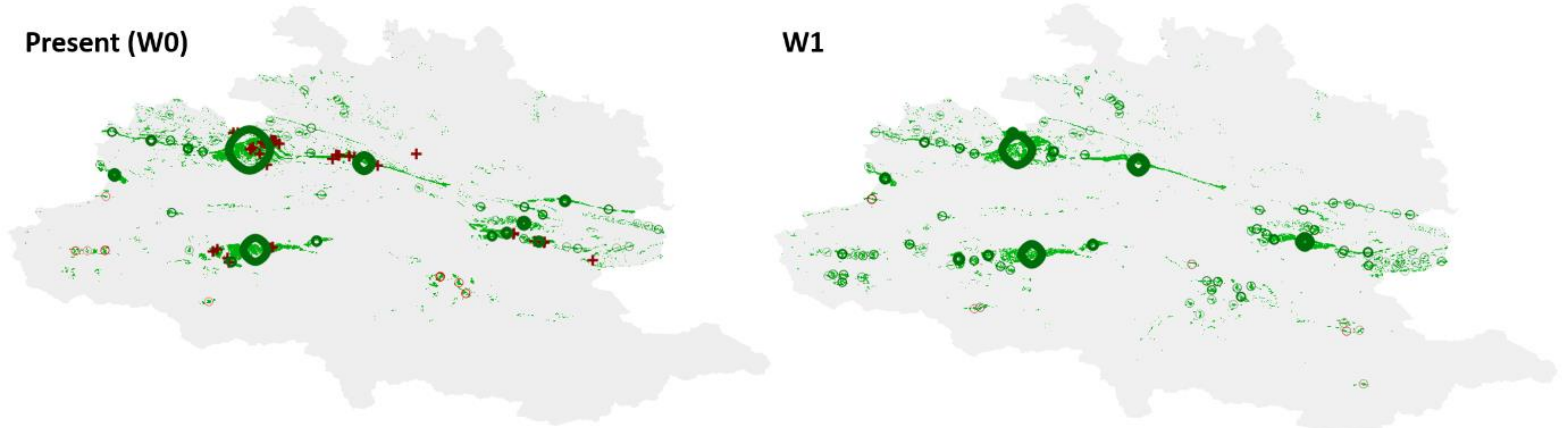

W2

W3
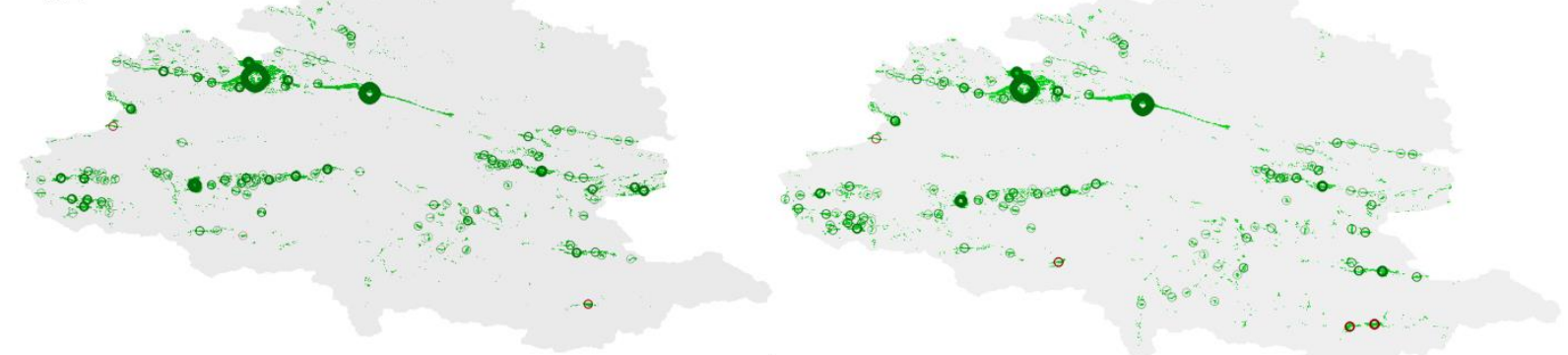

Fig.3 Map demonstrating suitable area (green, habitat suitability threshold $2 \times 10-3$ ) with reachable patch centroids (green circles) for the climate warming scenarios (ignoring ongoing habitat fragmentation). Climate warming scenarios are simulated by an increase in temperature of 1 to $3^{\circ} \mathrm{C}$ (W1-W3). The size of centroids is proportional to patch size. Red circles are predicted to be unreachable.

The Gentian distribution was mainly predicted based on geology (variable contribution to model: 81.5\%), followed by land use (12.5\%). April and July temperatures accounted for 0.4 and $2.7 \%$ of the relative Gentian presence probabilities, respectively (Fig.S7). The Alcon distribution was almost entirely predicted by Gentian presence probabilities (97.8\%) (Fig.S7). Excluding Gentian presence probabilities from the MAXENT model rendered land use (55.5\%) and July temperatures $(17.5 \%)$ the most important variables to predicted Alcon presences (Fig.S7).

The amount of suitable habitat is predicted to decrease with increasing temperatures (Fig.3), and patches steadily decrease in size and move upward as climate warms (Fig.3-4). However, connectivity is only slightly affected and no threatening situations occur even at a temperature increase of $3^{\circ} \mathrm{C}$ (Fig.3, Fig.4A left panel). Correspondingly, cooler temperatures reflecting past climate did not drastically affect Alcon distribution up to approximately $10 \mathrm{ky} B P\left(-2^{\circ} \mathrm{C}\right)$, and refugia may have been present at low altitude during the Last Glacial Maximum (Fig.S10). 

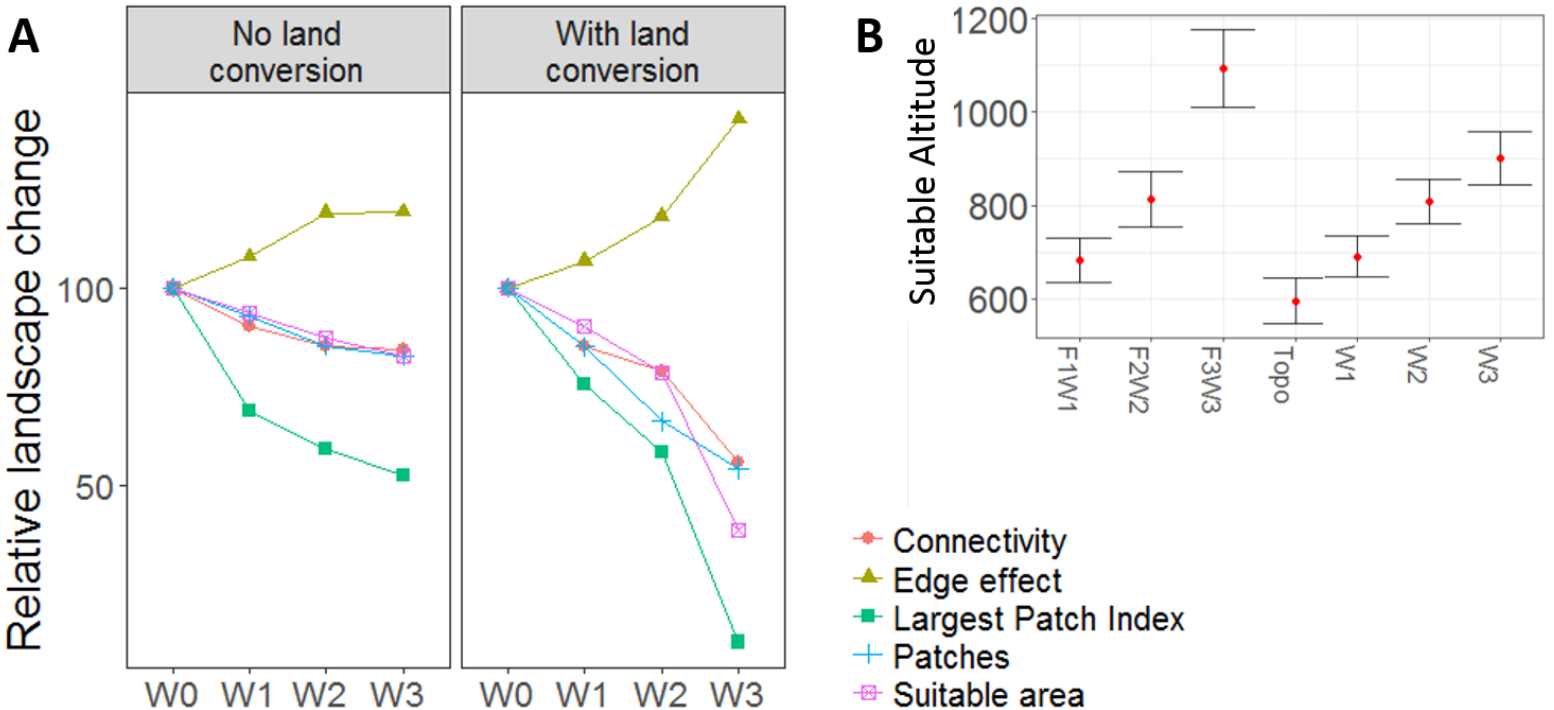

- Connectivity

- Edge effect

- Largest Patch Index

+ Patches

\& Suitable area

Fig.4. Effects of warming and land conversion on fragmentation levels as computed by FRAGSTATS (A) and on suitable patch altitude (B). W0 and Topo reflect current climate, W1 to W3 reflect climate warming by $1^{\circ} \mathrm{C}$ to $3^{\circ} \mathrm{C}$, and F1 to F3 reflect increasing levels of grassland conversion into forest.

Changes in land use drastically add to the negative impacts of climate warming on the predicted Alcon distribution (Fig.4-5). When temperature increases by $3^{\circ} \mathrm{C}$, and pastures and semi-natural grasslands become reforested, patches become too small and isolated for sustainable (meta)populations. Following expectations, abandonment of seasonal grazing practices is particularly detrimental for Alcon (F3W3, Fig.4-5). The suitable altitude doubles in this scenario (Fig.4). By the year 2100, suitable habitat, the number of suitable patches and connectivity are predicted to decrease by $25 \%, 32 \%$ and $32 \%$, resp. (Fig.6, Fig.S11). In addition, suitable habitat partially shifts to higher altitudes but low connectivity prevents dispersal to these higher patches (Fig.6). 
Present (FOW0)

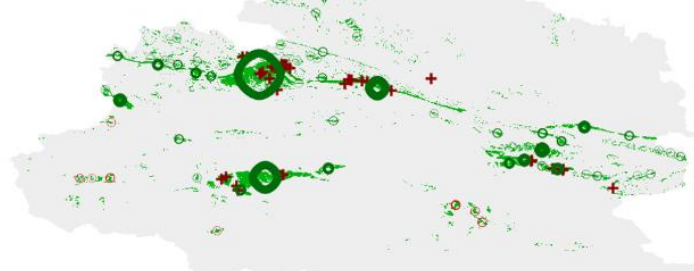

F2W2

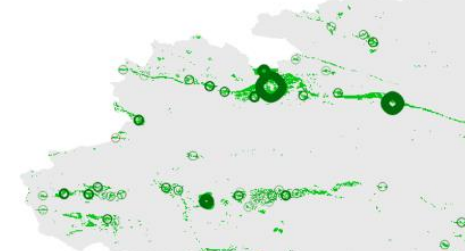

332

\section{F1W1}

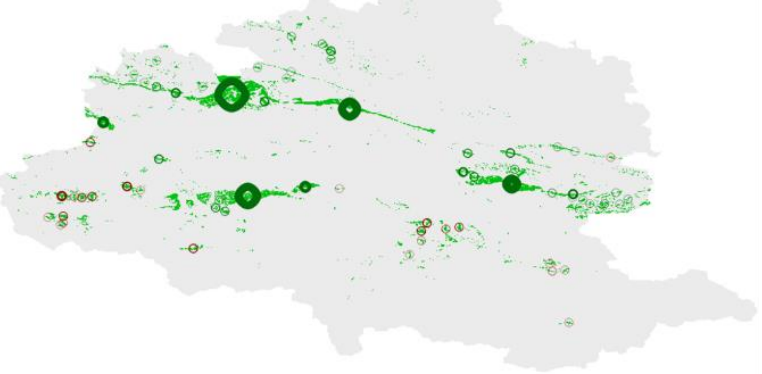

F3W3

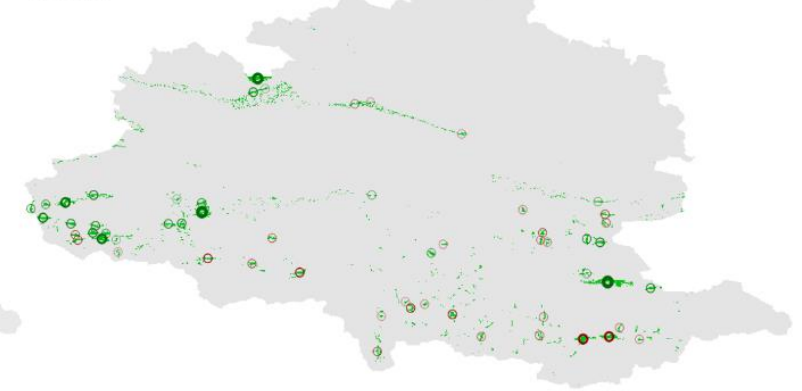

Fig.5. Maps demonstrating suitable area with patch centroids for the combined scenarios (accounting for ongoing habitat fragmentation). WO reflects current climate, W1 to W3 reflect climate warming by 1 to $3^{\circ} \mathrm{C}$, and F1 to F3 reflect increasing levels of grassland conversion into forest.

2100

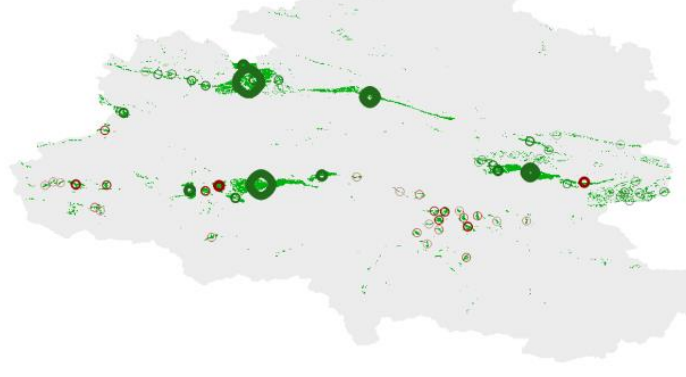

Fig.6. Maps demonstrating suitable area with patch centroids (left) and change in suitability as compared with the present (right) for the $\mathbf{2 1 0 0}$ scenario. Blue, green and red colors in the right panel represent suitable habitat gained, unchanged or lost, resp. Changes in fragmentation levels are also shown (legend following Fig.4).

Although in situ inspection of 30 checkpoint sites with promising predictability led to the discovery of 10 additional Alcon sites (from 27 to 37), these "New" sites are clustered near the same checkpoint site (Fig.7, Table S9). Moreover, this was the checkpoint with the lowest altitude (Fig.7). On average, the MAXENT habitat suitability was smallest in the recently extinct Alcon populations, followed by the checkpoint sites, and was highest in the existing and newly discovered sites (Fig.7). These differences 

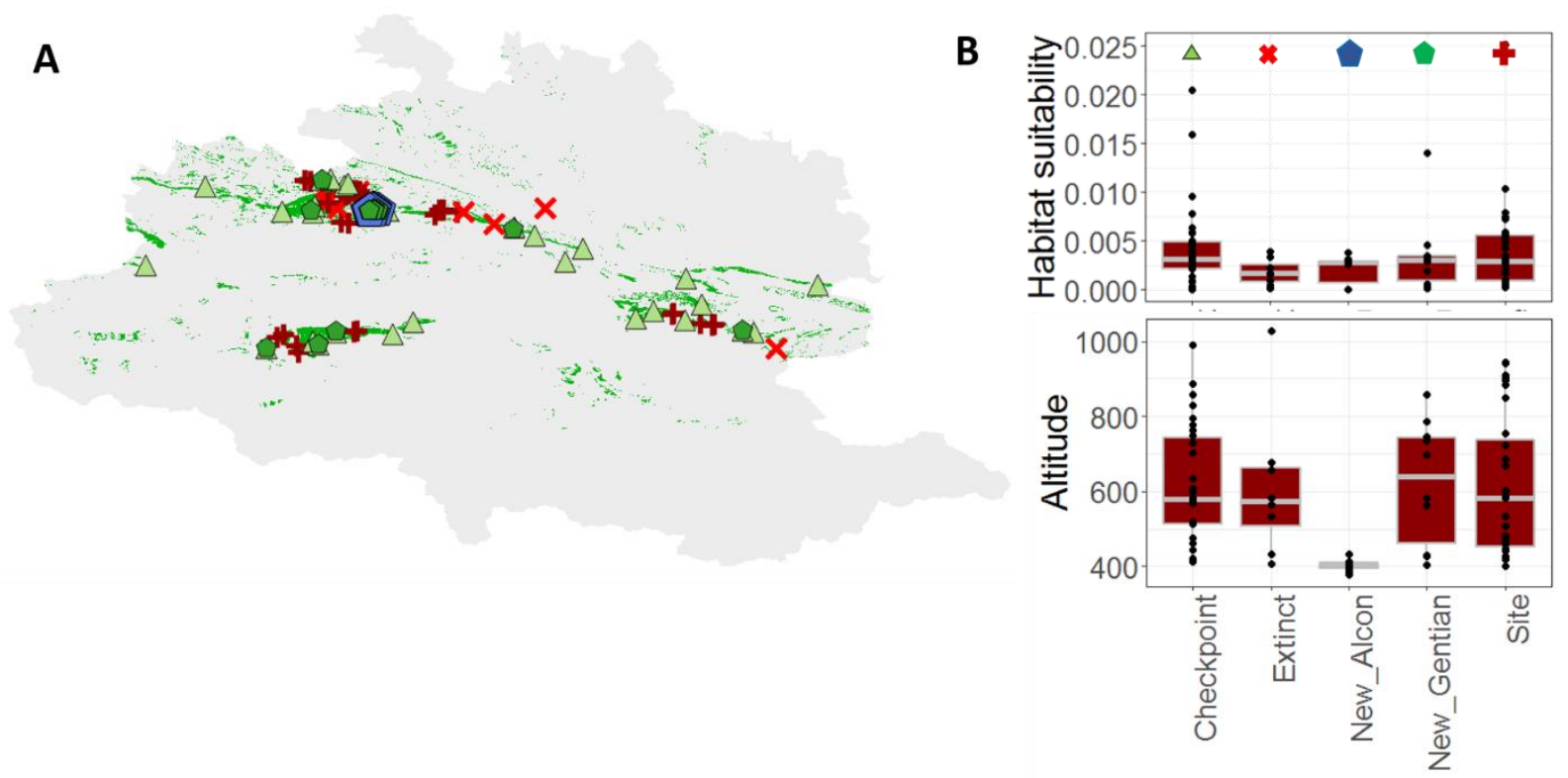

Fig.7. Position ( $A$ ), habitat suitability according to the TEMP ${ }_{\text {Topo }}$ scenario ( $B$, top) and altitude (B, bottom) for checkpoint sites, discovered sites near checkpoints ("New"), existing Alcon sites ("Site") and recently extinct Alcon sites. Panel B provides the legend for the symbols used in panel A.

Genetic diversity (GD) significantly decreased with increasing altitude ( $p<0.0001, R^{2}=0.65$, Fig.8). Neither local habitat suitability nor connectivity affected GD levels. Although Gentian GD did not add to the altitude effect, it was strongly correlated with both altitude and Alcon GD (Fig.8). Population sizes estimated from our CMR survey did not vary with altitude (Fig.S12), indicating that the genetic impoverishment with altitude probably resulted from a serial (upward) genetic founder effect followed by population expansion.
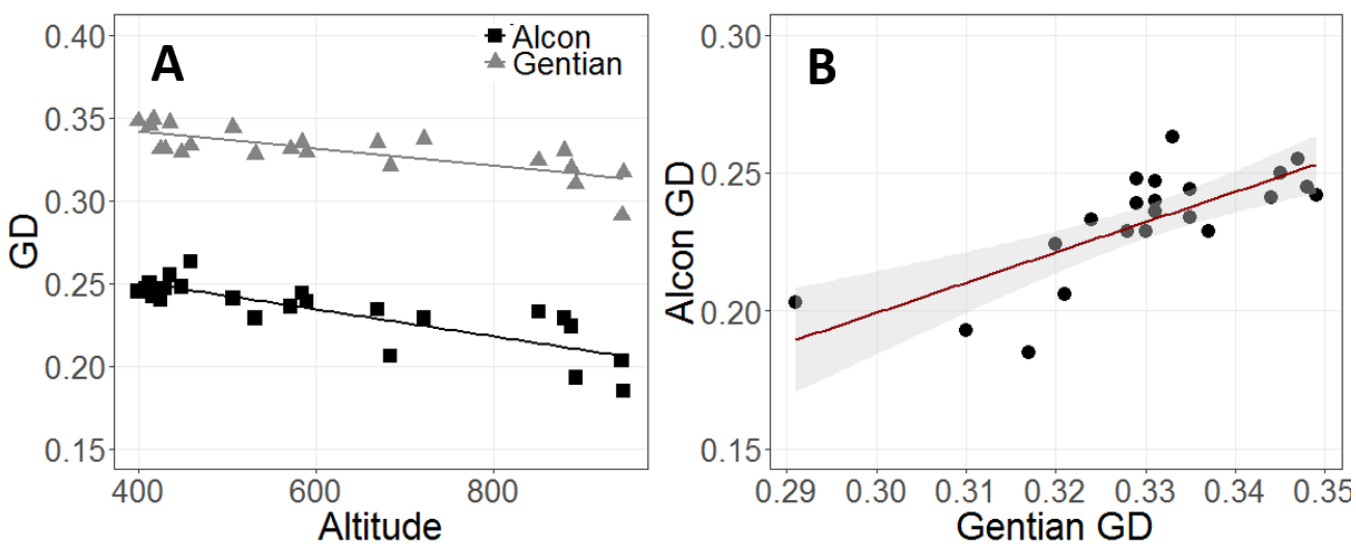

Fig.8. Relation between GD and altitude (A) and between Alcon and Gentian GD (B). 
The ability of species to cope with multiple global changes depends on their genetic diversity, dispersal abilities and interactions with the local biotic and abiotic environment. We show how climate and land use change notably push the expected distribution of a specialized grassland butterfly uphill and reduce the amount of habitat that is both suitable and reachable. Moreover, the marked correlation between genetic diversity and altitude suggests poor dispersal abilities of Alcon and its host plant, further challenging the future survival of both species. Our results suggest that many studies underestimate the impact of joint global environmental changes on community dynamics. On the other hand, conservation of semi-natural grasslands and woodland pastures may strongly mitigate the impacts of other global change drivers on Alcon butterflies and associated grassland species. In situ inspection of sites with promising presence probabilities was fruitful but demonstrated that our model still overestimates presence probabilities despite the inclusion of host plant distribution and microclimate.

\section{Impact of multiple environmental changes on a specialized grassland species}

Biotic interactions are fundamental to the functioning of natural communities and ecosystems, and shifts in their intensity and direction due to global environmental changes are triggering species' extinctions, community reorganization and disruption of ecosystem functioning (Gilman et al., 2010; Blois et al., 2013). Due to strong, often co-evolved interactions, specialist species are particularly vulnerable to changes in the distribution and abundances of their interacting counterpart. Assessing the contribution of biotic interactions to the evolutionary and demographic trajectories of specialist species therefore is indispensable to understand their ability to withstand multiple global changes and to develop targeted management strategies (Martin, 2001; Anderson, 2017). Our results show strong impacts of seasonal grazing abandonment on the estimated distribution of a specialized grassland butterfly, acting through the indirect effect on host plant availability (Fig.4-5). Also climate warming is expected to alter landscape configuration, mainly by reducing large suitable patches in size (Fig.3-4). Interestingly, parasitic species are by definition more rare than their host species, rendering the latter more suitable for predictive modelling. Our study correspondingly demonstrates how the prediction of host species' distribution in concert with field surveys and connectivity mapping can be used to identify core regions of suitable and reachable habitat for rare specialist species.

393 The implementation of biotic factors in ENM not only benefits predictions of specialist species, it also 394 has been proven useful with regard to generalist species (Sebastián-González et al., 2010; GonzálezSalazar et al., 2013). Using co-occurrence data for an insect genus (Lutzomyia sp.) and a mammal (Lynx rufus), Gonzales-Salazar et al. (2013) demonstrated that model predictability was higher for biotic variables than for abiotic variables, and maximized for both types of variables combined. Despite 
modelling has rarely been applied, and the development of community level ENM integrating population dynamics remains an important and poorly explored avenue in global change and conservation research (Thuiller et al., 2015; Anderson, 2017).

The field surveys associated with our study were kept manageable through the focus on a limited set of meta-communities, which allowed obtaining a better understanding of dispersal behaviour, a key aspect of predicting the realized niche under various ecological scenarios. This limited spatial scale and the particular cultural circumstances may, however, poorly represent distribution-wide survival prognoses. The variety in conservation and abandonment levels of mild grazing regimes across Eurasia implies that a range-wide integration of host presence, dispersal behaviour and abiotic factors are required to predict survival hot spots of Alcon and its host plant. Nevertheless, growing intensification of European farmlands (Sutcliffe et al., 2015) is expected to cause impacts on suitable patch size and connectivity comparable to reforestation of semi-natural grasslands.

Interestingly, climate warming increased levels of habitat fragmentation even without considering habitat fragmentation per se (Fig.3-4). Losses in suitable habitat due to temperature changes converts suitable into inhospitable habitat, thereby reducing patch size and connectivity, consequently compromising dispersal and gene flow. Moreover, habitat fragmentation per se reinforces suitable habitat shifts toward higher altitudes (Fig.4-5). Current alpine grasslands may therefore become (temporarily) suitable for Gentians and Alcons, and ideally become refugia during warmer periods as wild grazers systematically migrate upward.

\section{Genetically informed predictions and Conservation implications}

Genetic variation is key to long-term survival of populations across a species' range, as low genetic diversity jeopardizes local fitness and evolutionary potential (Reed \& Frankham, 2003; Carlson et al., 2014). Effective dispersal to suitable habitat therefore requires a sufficient amount of genetically diverse migrants that are able to found a sustainable population at a pace that equals or exceeds the velocity of environmental change (Davis \& Shaw, 2001; Corlett \& Westcott, 2013). We observed a signature of serial founder effects with altitude in Alcon and its host plant (Fig.8). Because population sizes did not decrease with altitude, this finding suggests that the species are recovering from a postglacial range shift toward higher altitudes without a marked fitness cost.

Although Alcon seems resilient to past and future temperature change (Fig.3, Fig.S8), the genetic founder effects and the limited dispersal observed here and in other studies (Vanden Broeck et al., 2017) imply that the species is expected to experience severe persistence difficulties due to increased levels of habitat fragmentation caused by (spontaneous) reforestation and climate warming. This also implies that ecological niche models ignoring reductions in habitat quality and quantity that are due to joint impacts of various types of global change may overestimate the predicted realized niche.

The lack of dispersal and gene flow between the northern and more isolated southern clusters is in line with the lower genetic diversity of the southern Alcon clusters (as opposed to the southern Gentian clusters) (Fig.S6). Possibly, Alcon is settling an extinction debt at the meta-population level, with 
observed levels of genetic diversity echoing formerly interconnected meta-populations during periods of more extensive grazing practices (see also Bulman et al., 2007; Habel et al., 2015; Fountain et al. 2016). The considerably longer generation time of Gentian and relatively high genetic diversity levels across the four meta-populations despite ongoing population declines across its range (Fig.S6) may imply a longer-term extinction debt where genetic diversity still fully reflects formerly interconnected meta-populations.

442 Ongoing declines in butterfly communities have frequently been attributed to losses of breeding areas 443 and increased isolation among them, rendering suitable patches less sustainable and poorly reachable 444 (Oliver et al., 2015; Thomas, 2016). Our findings are in line with this trend and suggest that habitat 445 fragmentation has more severe impacts than any other global environmental threat on specialized 446 grassland species. Reductions in connectivity, genetic diversity, fitness and/or in the potential to deal 447 with biotic (e.g. invasive species, disease) and abiotic (e.g. climate warming, extreme droughts) 448 changes render habitat fragmentation a critical enemy of biodiversity and ecosystem functioning 449 (Haddad et al., 2015; Newbold et al., 2015; Titeux et al., 2016). The conservation and restoration of 450 suitable and reachable habitat to protect the persistence and resilience of communities and 451 ecosystems may, however, be problematic in the Pyrenees, as the local causes of habitat 452 fragmentation are mainly driven by socio-economic factors. Ongoing rural depopulation of mountainous areas causes extensive grazing practices, traditionally referred to as seasonal 454 transhumance activities, to decrease, thereby facilitating spontaneous reforestation of suitable habitat 455 (Galop et al. 2011). This, combined with overgrazing of remaining grasslands, threatens many grassland specialist species in the Pyrenees (Galop et al. 2011). More rigorous management actions and

457 governmental interventions are required to mitigate the effects of land abandonment and 458 intensification on regional biodiversity. 
Anderson, R.P. (2017) When and how should biotic interactions be considered in models of species niches and distributions? Journal of Biogeography, 44,8-17.

462

463

464

465

466

467

468

469

470

471

472

473

474

475

476

477

478

479

480

481

482

483

484

485

486

487

488

489

490

491

492

493

494

Bailey, J.K., Genung, M.A., Ware, I., Gorman, C., Van Nuland, M.E., Long, H., \& Schweitzer, J.A. (2014) Indirect genetic effects: an evolutionary mechanism linking feedbacks, genotypic diversity and coadaptation in a climate change context. Functional Ecology, 28,87-95.

Blois, J.L., Zarnetske, P.L., Fitzpatrick, M.C., \& Finnegan, S. (2013) Climate Change and the Past, Present, and Future of Biotic Interactions. Science, 341,499-504.

Bulman, C.R., Wilson, R.J., Holt, A.R., Bravo, L.G., Early, R.I., Warren, M.S., Thomas, C.D. (2007) minimum viable metapopulation size, extinction debt, and the conservation of a declining species. Ecological Applications, 17(5),1460-1473.

Vanden Broeck, A., Maes, D., Kelager, A., Wynhoff, I., WallisDeVries, M., Nash, D., Oostermeijer, J., Van Dyck, H., \& Mergeay, J. (2017) Gene flow and effective population sizes of the butterfly Maculinea alcon in a highly fragmented, anthropogenic landscape. Biological Conservation, 209,89-79.

Carlson, S.M., Cunningham, C.J., \& Westley, P.A.H. (2014) Evolutionary rescue in a changing world. Trends in Ecology \& Evolution, 29,521-530.

Clavel, J., Julliard, R., \& Devictor, V. (2011) Worldwide decline of specialist species: toward a global functional homogenization? Frontiers in Ecology and the Environment, 9,222-228.

Collins, M., Knutti, R., Arblaster, J., et al. (2013) IPCC 2013. Long-term Climate Change Projections, Commitment and Irreversibility. Climate Change 2013: The physical science basis. Contribution of Working Group I to the Fifth Assessment Report of the Intergovernmental Panel on Climate Change. pp.1535.

Corlett, R.T. \& Westcott, D.A. (2013) Will plant movements keep up with climate change? Trends in Ecology \& Evolution, 28,482-488.

Davis, M.B. \& Shaw, R.G. (2001) Range shifts and adaptive responses to Quaternary climate change. Science, 292,673-9.

Eskildsen, A., Carvalheiro, L.G., Kissling, W.D., Biesmeijer, J.C., Schweiger, O., \& Høye, T.T. (2015) Ecological specialization matters: long-term trends in butterfly species richness and assemblage composition depend on multiple functional traits. Diversity and Distributions, 21,792-802.

Ettinger, A. \& HilleRisLambers, J. (2017) Competition and facilitation may lead to asymmetric range shift dynamics with climate change. Global Change Biology, 23,3921-3933.

Fernandez-Conradi, P., Jactel, H., Hampe, A., Leiva, M.J., \& Castagneyrol, B. (2017) The effect of tree genetic diversity on insect herbivory varies with insect abundance. Ecosphere, 8,e01637.

Fordham, D.A., Brook, B.W., Moritz, C., \& Nogués-Bravo, D. (2014) Better forecasts of range dynamics using genetic data. Trends in Ecology \& Evolution, 29,436-443.

Fountain, T., Nieminen, M., Sirén, J., Chong Wong, S., Lehtonen, R., Hanski, I. (2016) Predictable allele frequency changes due to habitat fragmentation in the Glanville fritillary butterfly. PNAS, 113(10),2678- 
Fridley, J.D. \& Grime, J.P. (2010) Community and ecosystem effects of intraspecific genetic diversity in grassland microcosms of varying species diversity. Ecology, 91,2272-2283.

Galop, D., Houet, T., Mazier, F., Leroux, G., Rius, D. (2011) Grazing activities and biodiversity history in the Pyrenees: New insights on high altitude ecosystems in the framework of a Human-Environment observatory. PAGES New, 19(2),52-55.

Gilman, S.E., Urban, M.C., Tewksbury, J., et al. (2010) A framework for community interactions under climate change. Trends in Ecology \& Evolution, 25,325-331.

Godsoe, W., Jankowski, J., Holt, R.D., \& Gravel, D. (2017) Integrating Biogeography with Contemporary Niche Theory. Trends in Ecology \& Evolution, 32,488-499.

González-Salazar, C., Stephens, C.R., \& Marquet, P.A. (2013) Comparing the relative contributions of biotic and abiotic factors as mediators of species' distributions. Ecological Modelling, 248,57-70.

Habel, J.C., Schmitt, T., Härdtle, W., Lütkepohl, M., Assmann, T. (2007) Dynamics in a butterfly-plant-ant system: influence of habitat characteristics on turnover rates of the endangered lycaenid Maculinea alcon. Ecological Entomology, 32 (5),536-543.

Habel, J.C., Brückmann, S.V., Schwarzer, J., Weig, A., Husemann, M., Steffan-Dewenter, N.I. (2015) Fragmentation genetics of the grassland butterfly Polyommatus coridon: Stable genetic diversity or extinction debt? Conservation Genetics, 16 (3),549-558.

Haddad, N.M., Brudvig, L.A., Clobert, J., et al. (2015) Habitat fragmentation and its lasting impact on Earth's ecosystems. Science Advances, 1,e1500052.

Halvorsen, R., Mazzoni, S., Bryn, A., Bakkestuen, V. (2015) Opportunities for improved species distribution modelling practice via a strict maximum likelihood interpretation of Maxent. Ecography, 38,172-183.

Hansen, M.C., Potapov, P. V., Moore, R., Hancher, M., Turubanova, S.A., Tyukavina, A., Thau, D., Stehman, S. V., Goetz, S.J., Loveland, T.R., Kommareddy, A., Egorov, A., Chini, L., Justice, C.O., \& Townshend, J.R.G. (2013) High-Resolution Global Maps of 21st-Century Forest Cover Change. Science, 342,850-853.

Hijmans, R.J., Cameron, S.E., Parra, J.L., Jones, P.G., \& Jarvis, A. (2005) Very high resolution interpolated climate surfaces for global land areas. International Journal of Climatology, 25,1965-1978.

Hill, N.J., Tobin, A.J., Reside, A.E., Pepperell, J.G., \& Bridge, T.C.L. (2016) Dynamic habitat suitability modelling reveals rapid poleward distribution shift in a mobile apex predator. Global Change Biology, 22,10861096.

Hughes, A.R., Inouye, B.D., Johnson, M.T.J., Underwood, N., \& Vellend, M. (2008) Ecological consequences of genetic diversity. Ecology letters, 11,609-23.

Ikeda, D.H., Max, T.L., Allan, G.J., Lau, M.K., Shuster, S.M., \& Whitham, T.G. (2017) Genetically informed ecological niche models improve climate change predictions. Global Change Biology, 23,164-176.

IUCN (World Conservation Union). 1990. Red list of threatened animals. World Conservation Monitoring Centre, Cambridge, UK. 
Kass J.M., Vilela B., Aiello-Lammens M.E., Muscarella R., Merow C., Anderson R.P. (2018) Wallace: A flexible platform for reproducible modelling of species niches and distributions built for community expansion. Methods in Ecology and Evolution doi.org/10.1111/2041-210X.12945.

Kittelson, P.M., Wagenius, S., Nielsen, R., Qazi, S., Howe, M., Kiefer, G., \& Shaw, R.G. (2015) How functional traits, herbivory, and genetic diversity interact in Echinacea : implications for fragmented populations. Ecology, 96,1877-1886.

Maes, D., Vanreusel, W., Talloen, W., Van Dyck, H. (2004) Functional conservation units for the endangered Alcon Blue butterfly Maculinea alcon in Belgium (Lepidoptera: Lycaenidae). Biological Conservation, 120 (2),229-241.

Martin, T.E. (2001) Abiotic vs. biotic influences on habitat selection of coexisting species: climate change impacts? Ecology, 82,175-188.

Mcgarigal, Cushman, S., Neel, M., \& Ene, E. (2002) FRAGSTATS: Spatial Pattern Analysis Program for Categorical Maps. Computer software program produced by the authors at the University of Massachusetts, Amherst.

McRae, B.H., Dickson, B.G., Roemer, G.W., \& Rundall, J.M. (2013) Circuitscape 4 User Guide. The Nature Conservancy. http://www.circuitscape.org.

Memmott, J., Craze, P.G., Waser, N.M., \& Price, M. V. (2007) Global warming and the disruption of plant?pollinator interactions. Ecology Letters, 10,710-717.

Merow, C., Smith, M.J., \& Silander, J.A. (2013) A practical guide to MaxEnt for modeling species' distributions: what it does, and why inputs and settings matter. Ecography, 36,1058-1069.

Merow, C., Silander Jr, J.A. (2014) A comparison of Maxlike and Maxent for modelling species distributions. Methods in Ecology and Evolution, 5,215-225.

Mouquet, N., Belrose, V., Thomas, J.A., Elmes, G.W., Clarke, R.T., \& Hochberg, M.E. (2005) Conserving community modules: A case study of the endangered lycaenid butterfly Maculinea alcon. Ecology, $86,3160-3173$.

Nash, D.R., Als, T.D., Maile, R., Jones, G.R., \& Boomsma, J.J. (2008) A Mosaic of Chemical Coevolution in a Large Blue Butterfly. Science, 319,88-90.

Nei, M., Maruyama, T., \& Chakraborty, R. (1975) The Bottleneck Effect and Genetic Variability in Populations. Evolution, 29,1-10.

Newbold, T., Hudson, L.N., Hill, S.L.L., et al. (2015) Global effects of land use on local terrestrial biodiversity. Nature, 520,45-50.

Oliver, T.H., Marshall, H.H., Morecroft, M.D., Brereton, T., Prudhomme, C., \& Huntingford, C. (2015) Interacting effects of climate change and habitat fragmentation on drought-sensitive butterflies. Nature Climate Change, 5,941-945.

Oostermeijer, J.G. , J.C.M. Dennijs, L.E.L. Raijmann, and S.B.J. Menken. (1992) Population biology and management of the marsh gentian (Gentiana pneumonanthe L), a rare species in the Netherlands. Botanical Journal of the Linnean Society, 108,117-130. 
Phillips, S.J. \& Dudík, M. (2008) Modeling of species distributions with Maxent: new extensions and a comprehensive evaluation. Ecography, 31,161-175.

Phillips, S.J., Dudík, M., \& Schapire, R.E. (2004) A maximum entropy approach to species distribution modeling. Proceeding, p83.

Prieto, I., Violle, C., Barre, P., et al. (2015) Complementary effects of species and genetic diversity on productivity and stability of sown grasslands. Nature Plants, 1,15033.

Queiroz, C., Beilin, R., Folke, C., \& Lindborg, R. (2014) Farmland abandonment: threat or opportunity for biodiversity conservation? A global review. Frontiers in Ecology and the Environment, 12,288-296.

Raes, N. \& ter Steege, H. (2007) A null-model for significance testing of presence-only species distribution models. Ecography, 30,727-736.

Reed, D.H. \& Frankham, R. (2003) Correlation between Fitness and Genetic Diversity. Conservation Biology, 17,230-237.

Sebastián-González, E., Sánchez-Zapata, J.A., Botella, F., \& Ovaskainen, O. (2010) Testing the heterospecific attraction hypothesis with time-series data on species co-occurrence. Proceedings of the Royal Society of London B: Biological Sciences, 277,DOI:10.1098/rspb.2010.0244.

Simmonds, M.W. (1946) Gentiana pneumonanthe L. Journal of Ecology, 33 (2),295-307.

Strona, G., Lafferty, K.D., Dunn, R.R., et al. (2016) Environmental change makes robust ecological networks fragile. Nature Communications, 7,12462.

Sutcliffe, L.M.E., Batáry, P., Kormann, U., et al. (2015) Harnessing the biodiversity value of Central and Eastern European farmland. Diversity and Distributions, 21,722-730.

Tessier, M. (2012) Inventaire de l'Azuré des mouillères Maculinea alcon (DENIS SCHIFFERMULLER, 1775) (Lepidoptera Lycaeniae) en Ariège. Bull. Soc. Linn. Bordeaux, 40,129-139.

Tessier, M. (2015) Inventaire de l'Azuré des mouillères Maculinea alcon (Denis \&amp; Schiffermüller, 1775) (Lepidoptera Lycaenidae) en Ariège. Bull. Soc. Linn. Bordeaux, 43,205-212.

Thomas, J.A. (2016) Butterfly communities under threat. Science, 353,216-218.

Thuiller, W., Pollock, L.J., Gueguen, M., \& Münkemüller, T. (2015) From species distributions to metacommunities. Ecology Letters, 18,1321-1328.

Titeux, N., Henle, K., Mihoub, J.-B., \& Brotons, L. (2016) Climate change distracts us from other threats to biodiversity. Frontiers in Ecology and the Environment, 14,291-291.

Tylianakis, J.M., Didham, R.K., Bascompte, J., \& Wardle, D.A. (2008) Global change and species interactions in terrestrial ecosystems. Ecology Letters, 11,1351-1363.

Vacquie, L.A., Houet, T., Sohl, T.L., Reker, R., \& Sayler, K.L. (2015) Modelling regional land change scenarios to assess land abandonment and reforestation dynamics in the Pyrenees (France). Journal of Mountain Science, 12,905-920.

WallisDeVries, M.F. (2004) A quantitative conservation approach for the endangered butterfly Maculinea alcon. 
604

605

606

607

608

609

610

611

612

613

614
Warren, M.S., Hill, J.K., Thomas, J.A., Asher, J., Fox, R., Huntley, B., Roy, D.B., Telfer, M.G., Jeffcoate, S., Harding, P., Jeffcoate, G., Willis, S.G., Greatorex-Davies, J.N., Moss, D., \& Thomas, C.D. (2001) Rapid responses of British butterflies to opposing forces of climate and habitat change. Nature, 414,65-69.

Weber, M.M., Stevens, R.D., Diniz-Filho, J.A.F., \& Grelle, C.E. V. (2017) Is there a correlation between abundance and environmental suitability derived from ecological niche modelling? A meta-analysis. Ecography, 40,817-828.

Wilmers, C.C. \& Post, E. (2006) Predicting the influence of wolf-provided carrion on scavenger community dynamics under climate change scenarios. Global Change Biology, 12,403-409.

Wynhoff, I. (1998) The recent distribution of the European Maculinea species. Journal of Insect Conservation, 2,15-27. 
616 The main research interests of the first author lie in exploring patterns of molecular diversity across

617 species' ranges, and in understanding population dynamics and environmental factors driving these 618 patterns. The other authors share this interest, with specific passion for dispersal dynamics (CT, MB 619 and VS), conservation (MT) and landscape genetics (JP). HDK wrote the manuscript, assisted with 620 sampling, and performed the lab work and data analyses. VS and MB provided the context of the 621 project and supervised. JGP performed the bio-informatics. MT located the community sites, 622 introduced the study system and visited checkpoint sites. All co-authors also commented on the 623 manuscript. 
624 SUPPORTING INFORMATION

625 Figures S1-S12 (pdf)

626 Tables S1-S8 (excel)

627 Map construction details (Appendix)

628 Sequencing details (Appendix) 